\title{
Anastomosis perpendicular duodeno-yeyunal para un acceso endoscópico convencional posoperatorio en las derivaciones biliodigestivas en Y de Roux
}

\author{
Perpendicular duodenum jejunum anastomosis for conventional postoperative \\ endoscopic access in biliodigestive diversions in Roux in $Y$
}

\author{
Arcenio L. Vargas-Ávila1, Guillermo Aguilera-Díaz-Teran', Julián Vargas-Flores, \\ Teresa de Jesús Galicia-Gómez ${ }^{1}$, Carlos A. Cortázar-Sánchez ${ }^{*}$, Arturo Cruz-Arias², \\ Miguel Camacho-Nájera ${ }^{3}$ y Ángel J. Lara-Valdez ${ }^{1}$ \\ ${ }^{1}$ Servicio de Cirugía General; ${ }^{2}$ Servicio de Imagenología Diagnóstica y Terapéutica; ${ }^{3}$ Servicio de Endoscopia Gastrointestinal. Hospital Regional \\ General Ignacio Zaragoza, Instituto de Seguridad y Servicios Sociales de los Trabajadores del Estado, Ciudad de México, México
}

\begin{abstract}
Resumen
Antecedentes: La colecistectomía es una de las cirugías más frecuentes, con aproximadamente 1.2 millones de procedimientos realizados anualmente solo en los Estados Unidos de América. La mayoría de las disrupciones biliares durante la colecistectomía laparoscópica se deben a una percepción errónea de la anatomía. Dos tercios de las estenosis posquirúrgicas se desarrollan 2-3 años después de la reparación y el otro tercio en los 10 años siguientes. Objetivo: Mostrar la adecuación para un acceso endoscópico posoperatorio en las derivaciones biliodigestivas en Y de Roux. Método: La mejor técnica quirúrgica reportada para la reparación de las disrupciones biliares es la anastomosis hepático-yeyuno o anastomosis colédoco-yeyuno en $Y$ de Roux. Partiendo de este procedimiento, la variante quirúrgica que proponemos consiste en realizar una anastomosis perpendicular entre el asa desfuncionalizada de yeyuno y el duodeno para el posterior acceso endoscópico de la derivación biliodigestiva en $Y$ de Roux. Resultados: Se realizó el procedimiento en cuatro pacientes con derivación biliodigestiva en $Y$ de Roux. Conclusiones: El procedimiento que proponemos tiene la ventaja de mantener abierta la anastomosis debido a su diseño romboidal, y como el píloro se encuentra indemne, el reflujo gástrico alcalino no sería ningún problema.
\end{abstract}

Palabras clave: Anastomosis. Perpendicular. Colédoco-yeyuno. Hepático-yeyuno.

\begin{abstract}
Background: Cholecystectomy is one of the most performed abdominal surgical procedures, with approximately 1.5 million procedures performed annually in the United States of america. Most of the biliary duct injuries during laparoscopic cholecystectomy are due to a wrong perception of the anatomy. Two thirds of postsurgical stenosis develop within two and three years after repairing and the other third in the following ten years. Objective: To show the adequacy for postoperative endoscopic access in Roux-in- $Y$ biliodigestive diversions. Method: The best technique to repair biliary duct injuries is the Roux-in- $Y$ hepaticojejunostomy or choledochojejunostomy, from this procedure we propose a surgical technique that consists to perform a perpendicular anastomosis between the defunctionalized jejunum loop and the duodenum for later endoscopic access of the
\end{abstract}

\section{Correspondencia:}

*Carlos A. Cortázar-Sánchez

Calz. Ignacio Zaragoza 1711

Ejército Constitucionalista, Chinam Pac de Juárez Fecha de recepción: 20-08-2020

C.P. 09220, Ciudad de México, México Fecha de aceptación: 25-10-2020

Cir Cir. 2021;89(5):638-645

E-mail: arturoc.csanchez@gmail.com

DOI: $10.24875 / C I R U .20000924$

Contents available at PubMed

www.cirugiaycirujanos.com

0009-7411/@ 2020 Academia Mexicana de Cirugía. Publicado por Permanyer. Este es un artículo open access bajo la licencia CC BY-NC-ND (http://creativecommons.org/licenses/by-nc-nd/4.0/). 
Roux-en-Y biliodigestive diversion. Results: The duodenal-jejunal perpendicular anastomosis procedure was performed in four patients with Roux-Y biliodigestive diversion. Conclusions: With the technique that is proposed, we have that advantage of keeping the anastomosis permanently open due to its rhomboidal design and because of the pylorus is kept intact alkaline reflux would not be a problem.

Key words: Anastomosis. Perpendicular. Hepaticojejunostomy. Choledochojejunostomy.

\section{Introducción}

La colecistectomía es una de las cirugías que más se realizan en todo el mundo, con aproximadamente 1.2 millones de procedimientos realizados anualmente solo en los Estados Unidos de América. Entre sus más graves complicaciones, la disrupción de la vía biliar se ha reportado en el $0.4-0.6 \%$ de las colecistectomías abiertas ${ }^{1}$, y el advenimiento de la colecistectomía laparoscópica ha conducido a un incremento en las tasas de disrupción de la vía biliar, con un rango del $0.3 \%$ al $0.6 \%{ }^{2}$, y con reportes de hasta el $1.4 \%{ }^{3}$ y el $1.5 \%{ }^{4}$. De estas, en torno al $17-20 \%$ son reconocidas intraoperatoriamente, con una marcada incidencia durante la curva de aprendizaje $^{5}$. La disrupción de la vía biliar después de la colecistectomía se define como una lesión no intencional de cualquier porción del tracto biliar que es evidenciado durante o después de la operación por fuga u obstrucción biliar ${ }^{4}$. Muchas de las disrupciones biliares durante la colecistectomía laparoscópica se deben a una percepción errónea de la anatomía más que a un inadecuado juicio o habilidad, lo que significa que las lesiones biliares continuarán a pesar de haber completado la curva de aprendizaje ${ }^{6}$.

Las estenosis biliares benignas son una condición relativamente rara que en la mayoría de los casos están causadas por un trauma quirúrgico, principalmente en una colecistectomía abierta o por vía laparoscópica, siendo la segunda causa la fibrosis en el sitio de anastomosis biliodigestiva ${ }^{7}$. La estenosis biliar se puede presentar en cualquier momento más allá de 6 semanas y hasta 15 años después de la disrupción biliar. La prevalencia del complejo atrofia/hipertrofia hepática después de las lesiones biliares es de aproximadamente el $10 \%$, con una prevalencia de cirrosis en estos pacientes del $4-17 \%{ }^{4}$.

La colangiografía transhepática percutánea se considera el método de referencia para el diagnóstico tardío de las lesiones, ya que además es una vía de descompresión en caso de colangitis, por lo que se recomienda previamente a la intervención quirúrgica. El otro estudio que provee información importante y tiene la ventaja de no ser invasivo es la colangiorresonancia ${ }^{6}$.

La sintomatología que estos pacientes presentan es muy variada, desde asintomáticos hasta una completa obstrucción con ictericia, dolor, prurito, litiasis biliar proximal, colestasis, abscesos intrahepáticos, colangitis y cirrosis biliar ${ }^{4,7}$. Se ha reportado una incidencia de colangiocarcinoma del $7.6 \%$ en los pacientes sometidos a coledocoduodenostomía y del $1.9 \%$ en los sometidos a hepatoyeyunostomía; este riesgo se incrementa en los pacientes que principalmente presentan colangitis en el seguimiento 4 .

El manejo de estas lesiones ha mostrado mejor resultado si son referidas a centros multidisciplinarios especializados en cirugía hepatobiliar ${ }^{2}$. Se ha reportado una completa rehabilitación después de una lesión biliar en el 75-98\% de los casos; este rango tan amplio de éxito puede explicarse por la diferencia en el mecanismo de la lesión y la variabilidad en el estado anatómico final de los conductos².

Con frecuencia, la extensión de la lesión isquémica sufrida por el conducto es menos evidente en la reparación inmediata, lo que resulta en una mayor incidencia de estenosis anastomóticas posoperatorias que requieren dilatación subsecuente o reparación quirúrgica, ya que las estenosis biliares se pueden manifestar tardíamente, incluso años después de la lesión ${ }^{5}$. Dos tercios de las estenosis posquirúrgicas se desarrollan 2-3 años después de la reparación y el otro tercio en los 10 años siguientes ${ }^{4}$. Se han reportado factores pronósticos desfavorables para el éxito de las reparaciones biliares, con menos éxito cuanto más alta sea la lesión, intentos de reparación temprana mientras el paciente curse con sepsis y peritonitis, lesiones vasculares asociadas y lesiones reparadas en centros sin experiencia. La reparación temprana tiene tal vez mejores resultados que la reparación tardía siempre y cuando sea realizada por un equipo especialista experimentado y bajo buenas condiciones del paciente ${ }^{4}$.

La mayoría de las lesiones se han resuelto realizando una anastomosis hepático-yeyuno en $Y$ de Roux $x^{2,5,7,8}$, 
por lo que puede considerarse la opción de reconstrucción de elección ${ }^{9,10}$. Realizando una anastomosis libre de tensión, amplia y con el adecuado material de sutura, en conductos de buena calidad y sin isquemia, se obtiene el mejor resultado ${ }^{10}$.

Todos estos procedimientos requieren un seguimiento periódico con la finalidad de evitar implicaciones medicolegales, por lo que estos pacientes deben ser sometidos a una vigilancia a largo plazo ${ }^{5}$.

La calidad de vida de los pacientes sometidos a derivaciones biliodigestivas deber ser evaluada hasta al menos 5 años después del procedimiento, para identificar fallas en el tratamiento, reestenosis 0 colangitis ${ }^{4}$.

Se han reportado diversos procedimientos para resolver la estenosis de la derivación biliodigestiva en Y de Roux, sobre todo en los casos con lesión de la vía biliar durante una colecistectomía o en algún otro caso en que la derivación en $Y$ de Roux fue el método de tratamiento. Cuando esta complicación grave se presenta, con la finalidad de tener un acceso posoperatorio de la estenosis, se han descrito varios métodos de tratamiento.

\section{Método}

Como es sabido, la mejor técnica quirúrgica reportada para la reparación de las lesiones biliares es la anastomosis hepático-yeyuno o colédoco-yeyuno en $Y$ de Roux $x^{4,5,7,8,11}$. Partiendo de este procedimiento, la variante quirúrgica que proponemos consiste en realizar una anastomosis perpendicular entre el asa desfuncionalizada de yeyuno, aproximadamente a $10-12 \mathrm{~cm}$ de distancia de la anastomosis bilio-yeyunal, y el duodeno, realizando una incisión duodenal en sentido longitudinal de $3.0 \mathrm{~cm}$ de longitud y otra incisión de la misma dimensión en sentido longitudinal del asa desfuncionalizada de yeyuno. Previamente ya se ha realizado la anastomosis bilio-yeyunal, quedando de esta forma ambas incisiones intestinales en posición perpendicular (Fig. 1), suturando la parte media del borde superior de la duodenotomía con el vértice superior de la yeyunotomía (Fig. 2), y a continuación el vértice proximal de la duodenotomía con la parte media del borde medial de la yeyunotomía (Fig. 3). Enseguida se sutura el vértice inferior de la incisión yeyunal con la parte medial del borde inferior de la duodenotomía y después se colocan puntos intercalados entre estos puntos de referencia, terminando la anastomosis con un punto en el vértice distal del

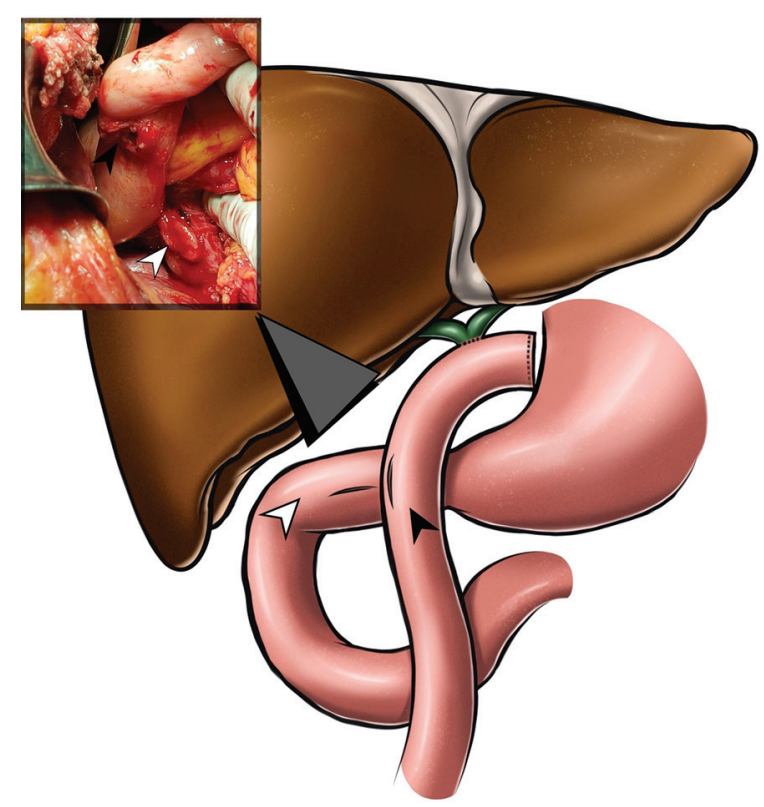

Figura 1. Derivación biliodigestiva colédoco-yeyuno. Punta de flecha blanca: incisión del duodeno. Punta de flecha negra: incisión del yeyuno.

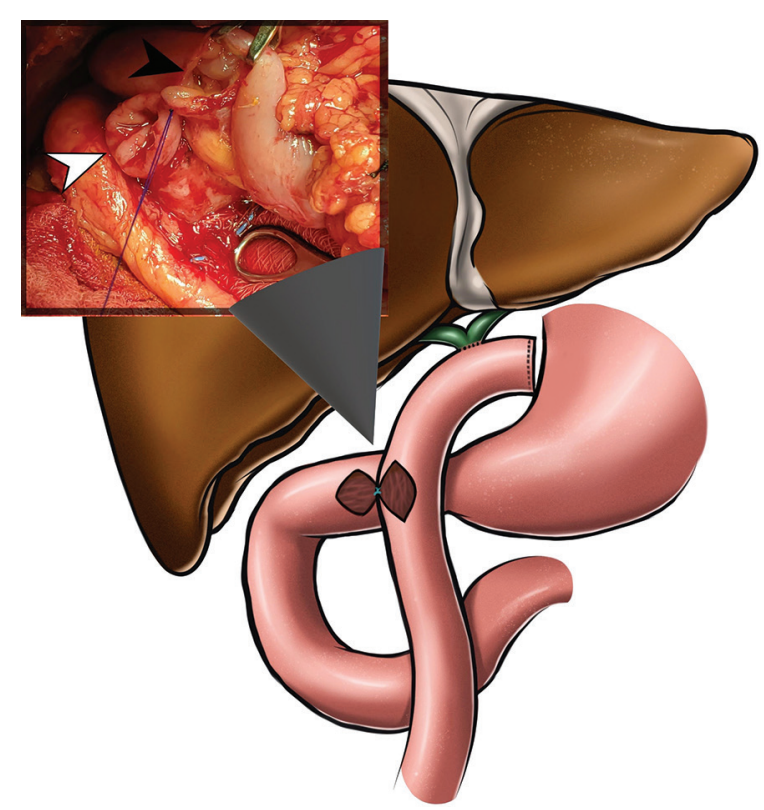

Figura 2. Derivación biliodigestiva colédoco-yeyuno. Punta de flecha negra: yeyuno. Punta de flecha blanca: duodeno.

duodeno con la parte media del borde lateral de la yeyunotomía, quedando de esta forma una anastomosis en forma de rombo (Figs. 4 y 5).

Este procedimiento ha sido diseñado para abordar las estenosis del sitio de anastomosis bilio-yeyunal por vía 


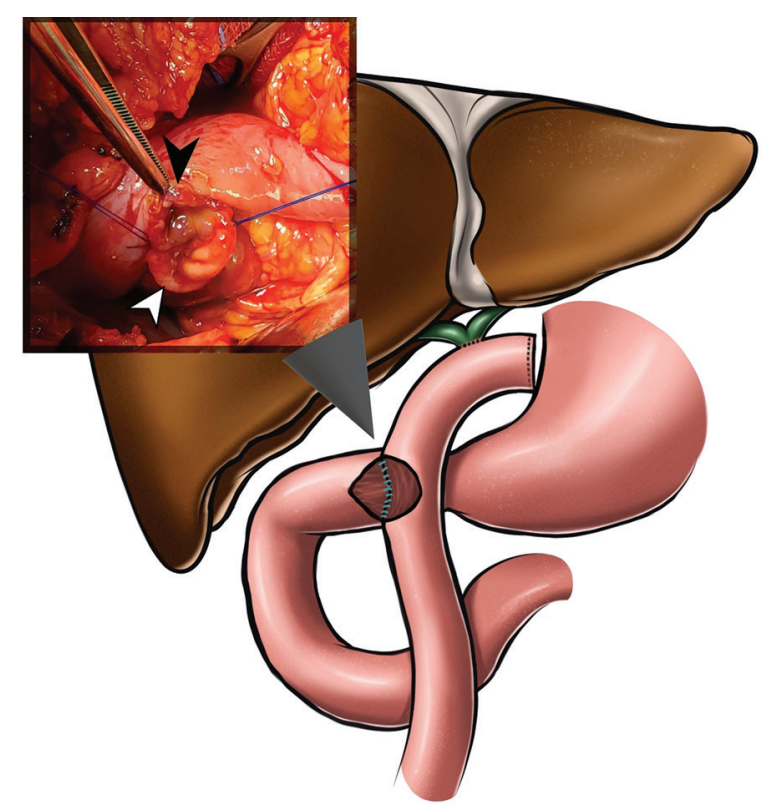

Figura 3. Derivación biliodigestiva colédoco-yeyuno. Punta de flecha negra: yeyuno. Punta de flecha blanca: duodeno.

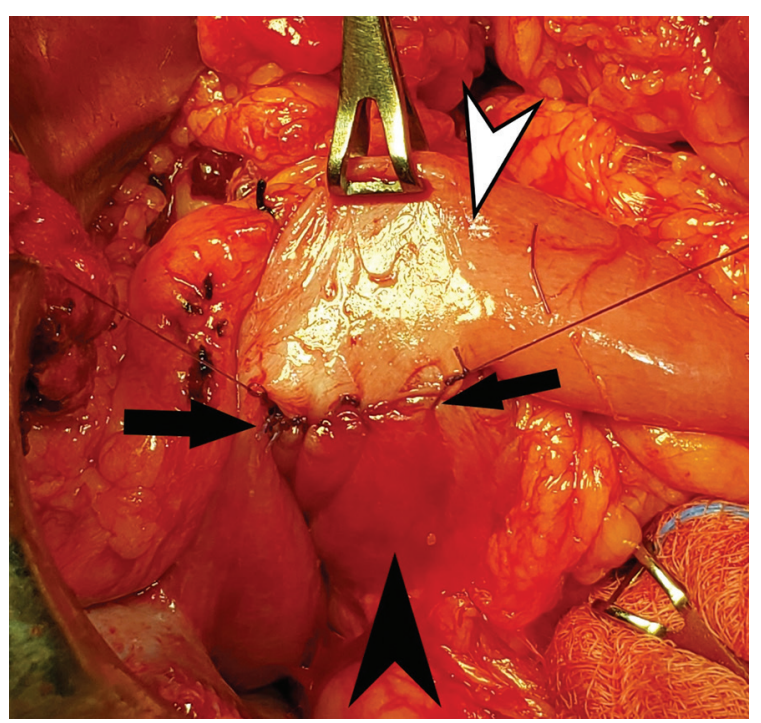

Figura 4. Anastomosis terminada. Punta de flecha blanca: yeyuno. Punta de flecha negra: duodeno. Flecha negra: anastomosis duodeno-yeyuno.

endoscópica convencional. Puede realizarse por método abierto o laparoscópico. El procedimiento fue aprobado por el comité de bioética de nuestro hospital.

\section{Resultados}

En noviembre de 2014 se realizó el primer procedimiento en fase experimental en cadáver, demostrando que era factible su realización.

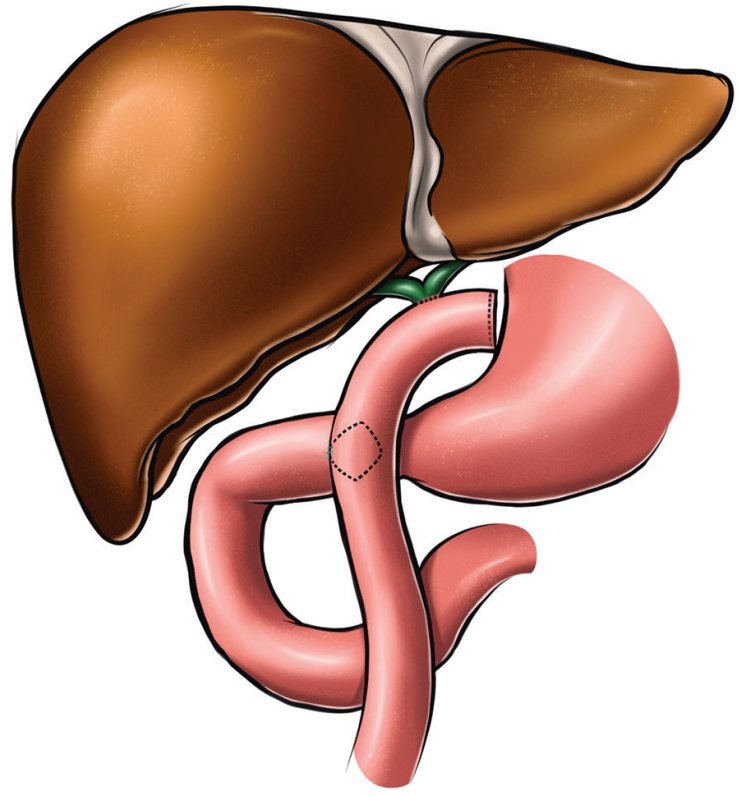

Figura 5. Anastomosis duodeno-yeyunal.

\section{Caso 1}

En noviembre de 2016 se llevó a cabo el primer procedimiento en un paciente vivo, cuyo diagnóstico preoperatorio fue el de quiste de colédoco tipo 1 de Todani, con evolución posoperatoria satisfactoria, mediante abordaje endoscópico de la anastomosis biliodigestiva 8 semanas después. Dicho abordaje fue realizado con panendoscopio y se demostró de esta forma que el abordaje de la anastomosis es factible con endoscopia convencional.

\section{Caso 2}

Se llevó a cabo en marzo de 2017 como complemento a una anastomosis hepático-yeyuno en $Y$ de Roux realizada 3 años antes. El paciente cursó con síndrome ictérico y colangitis de repetición, por lo que se le colocó un catéter para drenaje percutáneo temporal. Durante el procedimiento quirúrgico se abordó endoscópicamente a través de una incisión longitudinal del asa de yeyuno desfuncionalizada, logrando pasar el endoscopio, una guía y un globo para dilatación de la anastomosis estenosada y colocación de prótesis, y se finalizó el procedimiento con otra incisión longitudinal al eje duodenal y la anastomosis perpendicular duodeno-yeyunal en un plano. 


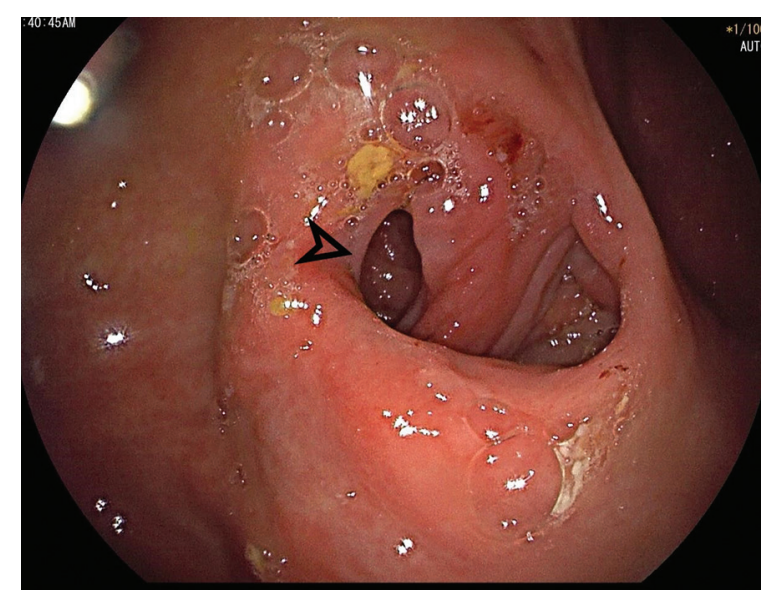

Figura 6. Vista endoscópica duodeno-yeyuno. Punta de flecha: sitio de entrada de la anastomosis duodeno-yeyuno.

\section{Caso 3}

En julio de 2019 se realizó el procedimiento a un paciente de 50 años con una lesión de vía biliar Bismuth 3 posterior a colecistectomía laparoscópica, que fue referido a nuestra unidad hospitalaria. En el mismo tiempo quirúrgico se le realizaron la derivación biliodigestiva y la anastomosis perpendicular duodeno-yeyunal (Fig. 6).

\section{Caso 4}

En agosto de 2019, a una paciente operada de derivación biliodigestiva en 2010 por lesión de la vía biliar en otra unidad hospitalaria. A lo largo de 9 años cursó con episodios recurrentes de colangitis y había sido sometida a ocho intervenciones transhepáticas percutáneas en los últimos 2 años. Se le realizó una laparotomía exploradora para anastomosis perpendicular duodeno-yeyunal, aprovechando la revisión endoscópica transoperatoria a través del yeyuno antes de realizar la anastomosis perpendicular duodeno-yeyunal, con hallazgo de cálculos dentro de conductos biliares, así como puntos de sutura al parecer de seda dentro de la luz a nivel de la anastomosis biliodigestiva.

\section{Discusión}

La anastomosis hepático-yeyuno es considerada el método de referencia para reconstruir las disrupciones biliares. Cuando la convergencia biliar es interrumpida, el conducto biliar izquierdo es aislado de acuerdo con la técnica de Hepp-Couinaud, y así ambos conductos, derecho e izquierdo, son anastomosados en la misma o en separadas aperturas intestinales ${ }^{4}$. Existen diversas clasificaciones de la lesión de la vía biliar, pero las más utilizadas son la de Bismuth y la de Strasberg ${ }^{12}$.

Entre las complicaciones tempranas de la anastomosis hepático-yeyuno en $Y$ de Roux, se produce fuga biliar con una frecuencia del 2.4-5.6 \% $\%^{8}$. Cuando esta fuga es persistente, es causada por uno o más conductos aislados que han pasado desapercibidos y no fueron anastomosados, para tratarla a veces es necesaria una inyección de etanol para esclerosar el conducto ${ }^{8}$.

La estenosis posoperatoria se reporta con una incidencia del $4-10 \%$, 13 , e incluso hasta del $25 \%$, y suele estar causada por una lesión quirúrgica del conducto biliar común durante una colecistectomía. La estenosis de la anastomosis es una seria complicación que puede conducir a episodios de colangitis de repetición, cirrosis biliar, falla hepática y muerte ${ }^{11,13-15}$. Así, un paciente que ha sido sometido a una simple cirugía de colecistectomía puede tornarse en un candidato a trasplante hepático debido a estenosis recurrente con desarrollo de cirrosis biliar ${ }^{16}$. La recurrencia de la estenosis se reporta en el $10 \%$ de los pacientes y requiere dilatación con balón y en algunos casos desmantelamiento y una nueva hepático-yeyunostomía ${ }^{6}$. La resolución de las estenosis a largo plazo, con cualquier tipo de terapia, todavía no es mayor del $84 \%$. Los factores de riesgo para la formación de estenosis son isquemia de los conductos biliares, múltiples intentos de reparación previa, abscesos intraabdominales o colecciones biliares, fístula biliar interna o externa, sexo masculino, anastomosis en un conducto no dilatado, drenaje percutáneo preoperatorio o posoperatorio, y comorbilidad que pueda comprometer la perfusión visceral13,17. Dentro de las complicaciones tardías se encuentran los eventos de colangitis, que a menudo se asocian a litiasis intrahepática, aunque usualmente se atribuyen a infección bacteriana retrógrada, por lo que es importante el diagnóstico de imagen para descartar una estenosis anastomótica8.

Luo, et al. ${ }^{18}$, para prevenir estenosis posoperatorias de las derivaciones biliodigestivas, reportan su procedimiento de dilatación progresiva con balón de la hepático-yeyunostomía con la colocación de una sonda en $T$ acompañada de balones en la anastomosis, 
abarcando los conductos derecho e izquierdo, con extracción de la rama larga de la sonda en T a través del asa de yeyuno por contra abertura, con dilatación progresiva subsecuente, reportando así mejores resultados en 52 de 54 pacientes que solo con la hepático-yeyunostomía.

La hepatectomía está indicada después de uno o varios intentos infructuosos de reparación, ya sea por vía endoscópica o quirúrgica, y en presencia de sepsis secundaria ${ }^{4}$.

Barbier, et al. ${ }^{4}$ reportan el trasplante hepático como una indicación excepcional y reservada para pacientes cuya función hepática está tan deteriorada que una reparación o hepatectomía son imposibles después de una lesión vascular biliar grave y lesiones biliares en pacientes con enfermedad hepática preexistente ${ }^{4}$.

Parrilla, et al. ${ }^{19}$, en España, realizaron un estudio retrospectivo y multicéntrico, de enero de 1987 a diciembre de 2010, en el que se incluyeron 27 pacientes en lista de espera para trasplante después de haber sido sometidos a uno o varios intentos de reparación de lesiones biliares, y concluyeron que las lesiones en la colecistectomía laparoscópica son más graves que en la cirugía abierta.

El objetivo del tratamiento de las estenosis biliares posoperatorias es establecer el flujo biliar hacia el tracto gastrointestinal, de manera que se pueda prevenir la aparición de colestasis, colangitis, lodo o cálculos $^{14}$. Este objetivo se logra suturando los tejidos sanos y realizando anastomosis libres de tensión ${ }^{2}$. Se han propuesto varios métodos, incluyendo anastomosis termino-terminales, colédoco-duodenostomías, hepático-yeyunostomía y colédoco-yeyunostomías en $Y$ de Roux o interposición de injertos mucosos, entre otros $^{14}$.

Dentro de los procedimientos para abordar las estenosis posoperatorias se incluyen técnicas no quirúrgicas, como la endoscópica percutánea usando balones para dilatación o la colocación de endoprótesis ${ }^{2,7,13}$. De Reuver, et al. ${ }^{3}$ reportan el manejo endoscópico con endoprótesis con una tasa de éxito para las estenosis biliares por lesión biliar del $74 \%$, con una duración del tratamiento con stents de 11 meses, todos con un seguimiento de 4.5 años, aunque estos resultados no han sido reproducidos de forma homogénea en otros reportes.

Existen también técnicas quirúrgicas, como la reanastomosis de la anastomosis hepático-yeyuno, que parece ser el método quirúrgico más común. Así, Stilling, et al. ${ }^{16}$, en Dinamarca, reportan que al menos el $42 \%$ de los pacientes presentaron una complicación a largo plazo, siendo la más frecuente la colangitis, la cual ocurrió en el $23 \%$ de ellos, y el $30 \%$ presentó estenosis de la anastomosis hepático-yeyuno. Además, en su serie reportan lesión vascular en el 19\% de los casos. Lo que podría explicar la alta incidencia de estenosis es que en éste grupo los pacientes fueron sometidos tempranamente a la reconstrucción biliar, el $83 \%$ de ellos dentro de las primeras 2 semanas después de la lesión y los restantes dentro de los primeros 2 días.

Se ha utilizado una gran variedad de procedimientos quirúrgicos con asas de acceso biliar con la finalidad de facilitar el intervencionismo endoscópico, para lo cual muchos autores se han dado a la tarea de diseñar una serie de procedimientos. Barker y Winkler ${ }^{20}$ publicaron un procedimiento adicional a la hepático-yeyunostomía, llevando la parte terminal del asa proximal de la $Y$ de Roux a la pared abdominal, construyendo un estoma de yeyuno en el área subcostal derecha de la pared abdominal. Helmy, et al. ${ }^{9,21}$ reportan el uso de un tubo gástrico pediculado interpuesto entre los segmentos proximal y distal de la vía biliar lesionada, en un primer caso clínico, con buenos resultados en un seguimiento de 5 meses, y una serie de 18 casos con lesiones y pérdida de segmento con un seguimiento a 2 años sin complicaciones. Consideran que tiene ventajas sobre otros métodos porque se preserva el esfínter de Oddi funcional, facilitando procedimientos diagnósticos y terapéuticos como la colangiopancreatografía retrógrada endoscópica, la cual ha probado ser sumamente difícil después de una anastomosis hepático-yeyuno en $Y$ de Roux. Otra opción es conectar un tubo gástrico interpuesto al duodeno; recordemos que el tubo gástrico tiene epitelio mucoso superficial, el cual, al estar en contacto continuo con la bilis, sufriría inflamación de origen alcalino. Crema, et al. ${ }^{22}$ reportan sus primeros casos de retubularizacion con yeyuno pediculado interpuesto entre el conducto biliar y el duodeno.

Elbir, et al. ${ }^{13}$ reportan el caso de un paciente tratado con plastia de la zona de estenosis utilizando los principios de la técnica de Heineke-Mikulicz, con una buena evolución en un seguimiento de 18 meses, pero en caso de reestenosis este paciente tendría que someterse a una nueva exploración quirúrgica o bien por abordaje percutáneo, con un resultado incierto.

El manejo endoscópico es el método menos invasivo, pero su principal obstáculo es el acceso extremadamente difícil del endoscopio al shunt bilioentérico debido a la anatomía alterada de la reconstrucción en $Y$ de Roux, aunque algunos autores defienden el uso 
de la enteroscopia con balón único o doble para superar esta dificultad ${ }^{11}$.

Otros autores construyen asas quirúrgicas de acceso para que el endoscopista alcance el sitio de la hepático-yeyunostomía. Estas asas pueden ser de yeyuno cutáneas superficial o subfacial, hepático-yeyunal subparietal, descrita por Beckingham, et al. ${ }^{23}$, donde el asa desfuncionalizada se cierra y se fija en posición subparietal a la pared abdominal, con ligaclips colocados para marcar la anastomosis con el fin de una identificación radiológica posterior, (fluoroscópicamente determinando la posición del 'Ligaclip'), el bucle se introduce por vía percutánea entre los Clips y utilizan una aguja de calibre fino con intermitentes inyecciones de contraste y una guía. Reportan el uso del asa larga desfuncionalizada de yeyuno (el extremo del asa de $Y$ de Roux que se toma para la hepaticoyeyunostomia) colocada en posición retrocólica y subcutánea como si fuera una yeyunostomía de alimentación pero con válvula para evitar el reflujo ${ }^{20} \mathrm{O}$ asas de yeyuno con acceso gástrico (yeyunogastrostomía), como el procedimiento reportado por Sitaram, et al. ${ }^{24}$ en su serie.

Hamad, et al. ${ }^{11}$ evaluaron el uso de tres diferentes técnicas de bilioenterogastrostomía, la cual consiste en la elaboración de un shunt bilioentérico modificado con un asa de acceso gástrico, para un acceso endoscópico a la hepático-yeyunostomía y de esta forma manejar la estenosis posquirúrgica, pero presenta como desventaja su complejidad y el efecto adverso de la bilis sobre el epitelio gástrico.

Con la técnica que nosotros proponemos se tiene la ventaja de mantener abierta en forma permanente la anastomosis debido a su diseño romboidal, y como el asa de yeyuno se encuentra desfuncionalizada y el píloro está indemne, el reflujo gástrico alcalino no es ningún problema. El procedimiento propuesto se puede realizar durante la misma derivación biliodigestiva o bien como en los casos 2 y 4 que presentamos, en los que se adicionó la anastomosis perpendicular duodeno-yeyunal en el posoperatorio tardío.

\section{Conclusiones}

Ninguna de las opciones terapéuticas para abordar los sitios de anastomosis hepático-yeyunal en $Y$ de Roux, en estado posoperatorio, ha sido cien por ciento efectiva, por lo que se han diseñado diversas técnicas con sus variantes, con tal de acceder el sitio remoto de la anastomosis, cuando ha ocurrido una estenosis de esta, como una de las más temidas complicaciones, por la morbilidad y las secuelas que conlleva en caso de no ser resuelta. Con la técnica de anastomosis perpendicular duodeno-yeyunal, adicional a la hepático-yeyunostomía que aquí presentamos, es factible abordar en forma directa el sitio de anastomosis con endoscopía convencional, y por su diseño permite la repetición a demanda para mantener la permeabilidad y la funcionalidad a largo plazo.

\section{Agradecimientos}

Al servicio de radiología e imagen y de endoscopia gastrointestinal del Hospital Regional General Ignacio Zaragoza.

\section{Responsabilidades éticas}

Protección de personas y animales. Los autores declaran que los procedimientos seguidos se conformaron a las normas éticas del comité de experimentación humana responsable y de acuerdo con la Asociación Médica Mundial y la Declaración de Helsinki.

Confidencialidad de los datos. Los autores declaran que han seguido los protocolos de su centro de trabajo sobre la publicación de datos de pacientes.

Derecho a la privacidad y consentimiento informado. Los autores han obtenido el consentimiento informado de los pacientes y/o sujetos referidos en el artículo. Este documento obra en poder del autor de correspondencia.

\section{Conflicto de intereses}

Los autores declaran no tener conflictos de intereses.

\section{Bibliografía}

1. Zhi VF, Henry AP, Strasberg SM, Loehrer AP, Sicklick JK, Talamini MA, et al. Diminished survival in patients with bile leak and ductal injury: management strategy and outcomes. J Am Coll Surg. 2018;226:568-76.

2. Mercado MA, Franssen B, Domínguez I, Arriola-Cabrera JC, Ramírez-Del Val F, Elnecavé-Olaiz A, et al. Transition from a low- to high-volume centre for bile duct repair: changes in technique and improved outcome. HPB. 2011;13:767-73.

3. De Reuver PR, Rauws EA, Vermeulen M, Dijkgraaf MGW, Gouma DJ, Bruno MJ. Endoscopic treatment of post-surgical bile duct injuries: long term outcome and predictors of success. Gut. 2007;56:1599-605.

4. Barbier L, Souche R, Slim K, Ah-Soune P. Long-term consequenses of bile duct injury after cholecystectomy. J Visc Surg. 2014;151:269-79.

5. Silva MA, Coldham C, Mayer AD, Bramhall SR, Buckels JAC, Mirza DF. Specialist outreach service for on-table repair of iatrogenic bile duct injuries - a new kind of travelling surgeon. An R Coll Surg Engl. 2008;90:343-6.

6. Sankar S, Subramanian M. Laparoscopic bile duct injuries - controversies and consensus. Sri Ramachandra J Med. 2007;1(2).

7. Huszár $O$, Kokas $B$, Mátrai $P$, Hegyi $P$, Pétervári $E$, Vincze $A$, et al Meta-analysis of the long-term success rate of different interventions in benign biliary strictures. PLoS One. 2017;12:e0169618. 
8. Hirano S, Tanaka E, Tsuchikawa T, Matsumoto J, Shichinohe T, Kato K Techniques of biliary reconstruction following bile duct resection. $\mathrm{J} \mathrm{He}$ patobil Pancreat Sci. 2012;19:203-9.

9. Helmy AA, Hamad MA, Aly AM, Sherif T, Hashem M, El-Sers DAH, et al Novel technique for biliary reconstruction using an isolated gastric tube with vascularized pedicle: a live animal experimental study and the first clinical case. Ann Surg Innov Res. 2011;5:8.

10. Mercado MA, Arriola JC, Domínguez I, Elnecavé Olaiz A, Urencio $M$, Ramírez del Val $F$, et al. Lesión iatrogénica de vía biliar con pérdida de confluencia: opciones quirúrgicas. Cir Gen. 2010 $32(3)$

11. Hamad MA, El-Amin H. Bilio-entero-gastrostomy: prospective assessment of a modified biliary reconstruction with facilitated future endoscopic access. BMC Surg. 2012;12:9.

12. Lubikowski J, Piotouch B, Stadnik A, Przedniczek M, Remiszewski P Milkiewicz $P$, et al. Difficult iatrogenic bile duct injuries following different types of upper abdominal surgery: report of three cases and review of literatura. BMC Surg. 2019;19:162.

13. Elbir OH, Karaman K, Surmelioglu A, Bostanci EB, Akoglu M. The Heineke-Mickulicz principle for hepaticoyeyunostomy stricture. Case Rep Surg. 2012;2012:454975.

14. Tocchi A, Costa G, Lepre L, Liotta G, Mazzoni G, Sita A. The long-term outcome of hepaticoyeyunostomy in the treatment of benign bile duct strictures. Ann Surg. 1996;224:162-7.

15. Ramesh H, Prakash K, Kuruvilla K, Philip M, Jacob G, Venugopal B, et al. Biliary access loops for intrahepatic stones: results of jejunoduodenal anastomosis. ANZ J Surg. 2003;73:306-12.
16. Stilling NM, Fristrup C, Wettergren A, Ugianskis A, Nygaard J, Holte K, et al. Long-term outcome after early repair of iatrogenic bile duct injury. A National Danish Multicentre study. HPB. 2015;17:394-400.

17. Booij KAC, Coelen RJ, de Reuver PR, Basselink MG, van Delden OM, Rauws EA, et al. Long-term follow-up and risk factors for strictures after hepaticojejunostomy for bile duct injury: an analysis of surgical and percutaneous treatment in a tertiary center. Surgery. 2018;163:1121-7.

18. Luo ZL, Cheng L, Ren JD, Tang LJ, Wang T, Tian FZ. Progressive balloon dilatation following hepaticojejunostomy improves outcome of bile duct stricture after iatrogenic biliary injury. BMC Gastroenterol. 2013;13:70.

19. Parrilla $P$, Robles $R$, Varo $E$, Jiménez $C$, Sánchez-Cabús $S$, Pareja $E$. Liver transplantation for bile duct injury after open and laparoscopic cholecystectomy. BJS. 2014;101:63-8.

20. Barker EM, Winkler M. Permanent-access hepaticojejunostomy. $\mathrm{Br} J$ Surg. 1984;71:188-91.

21. Helmy AA, Ali AMA. latrogenic bile duct injury repair using isolated vascularized gastric tube: early experience in two tertiary centers. Int Surg J. 2017:4:1825-32.

22. Crema E, Trentini EA, Teles CJ, Monti PR, Lacerda CF, Junior JA, et al. Laparoscopic reconstruction of the extrahepatic bile duct using a jejunal tuve: an innovative, more physiological and anatomical technique for biliodigestive derivation. J Surg Case Rep. 2014;2014:rjt106.

23. Beckingham IJ, Krige JEJ, Beningfield SJ, Bornman PC, Terblanche J. Subparietal hepaticojejunal access loop for the long-term management of intrahepatic stones. Br J Surg. 1998;85:1360-3.

24. Sitaram V, Perakath B, Chacko A, Ramakrishna BS, Kurian G, Khanduri P. Gastric access loop in hepaticojejunostomy. Br J Surg. 1998;85:108-10. 